\title{
Modelo español de MEDEVAC. Experiencia en Afganistán
}

\author{
Munayco Sánchez AJ. ${ }^{1}$, Navarro Suay R. ${ }^{2}$, de Nicolás Álvarez MA. ${ }^{1}$
}

Sanid. mil. 2012; 68 (3): 182-184; ISSN: 1887-8571

\section{RESUMEN}

El presente informe trata de desarrollar la experiencia acumulada por los equipos de Evacuación Médica (MEDEVAC), entre los años 2005 y 2010, desde el despliegue del ROLE 2 en la Base de Herat, Afganistán, contando con función de aeroevacuación avanzada y táctica. Describimos las aeronaves utilizadas, el componente sanitario de las tripulaciones y las habilidades asistenciales practicadas además de exponer iconografía resumen de las evacuaciones realizadas y su casuística. Finalmente describimos un resumen final y perspectivas de futuro.

PALABRAS CLAVE: ISAF, MEDEVAC, Aeroevacuación, Hospital ROLE 2.

\section{Spanish MEDEVAC system: The Afghanistan Experience}

\section{SUMMARY}

The purpose of this report is to show the experience gathered by the Medical Evacuation (MEDEVAC) teams between 2005 and 2010 , during the deployment of the ROLE 2 in Herat Base, Afghanistan, carrying out tactical and advanced aeromedical evacuation. Deployed aircrafts, medical crew and the practised clinical skills are described, as well as iconography is shown to summarize the performed evacuations and their casuistics. Finally, a summary and future prospects are presented.

KEY WORDS: ISAF, MEDEVAC, Aeromedical evacuation, ROLE 2.

\section{INTRODUCCIÓN}

Desde el año 2005 el Ejército del Aire contribuye dentro de la misión «Fuerza para la Asistencia en la Seguridad Internacional» (ISAF) en Afganistán con varios apoyos, entre los que cabe reseñar el despliegue de un hospital ROLE 2, actualmente ROLE 2 mejorado (ROLE 2E) con capacidad MEDEVAC. El objeto de este informe se centra en las aeroevacuaciones avanzadas y tácticas. En las avanzadas el herido es trasladado desde el punto de impacto hasta el escalón sanitario útil más cercano. Generalmente estas evacuaciones se efectúan mediante aeronaves de ala rotatoria. En las aeroevacuaciones tácticas el herido es trasladado intrateatro entre diferentes escalones sanitarios. Para este fin se utilizan medios de ala rotatoria o fija ${ }^{2}$.

En ambos tipos de evacuaciones el modelo español apuesta por una tripulación sanitaria completa, es decir, compuesta por médico, enfermero y sanitario capaces de aplicar soporte vital avanzado desde el inicio de la asistencia ${ }^{3}$. Esto debe incidir positivamente en el pronóstico de las bajas trasportadas.

La aplicación de técnicas avanzadas en las condiciones más duras, exige formación actualizada y revalidada para todos los componentes de la tripulación sanitaria, además del compromiso firme de ser capaces de incorporar a su práctica asistencial aquellas nuevas técnicas y tecnologías de eficacia contrastada y desarrollar otras propias de acuerdo a la experiencia ${ }^{4}$.

${ }^{1}$ Cap. Médico. Unidad Médica Aérea de Apoyo al Despliegue (UMAAD). Madrid. España. ${ }^{2}$ Capitán Médico. Escuela Militar de Sanidad. Madrid. España.

Dirección para correspondencia: Armando José Munayco Sánchez. Base Aérea de Torrejón. 28850 Torrejón de Ardoz (Madrid). E mail: delascuevs@hotmail.com

Recibido: 14 de marzo de 2011

Aceptado: 7 de mayo de 2012
Se deben contemplar previamente las diferentes configuraciones posibles de las aeronaves adaptadas al número y tipo de heridos, así como al modelo de aeronave utilizada junto con las condiciones y distancia de vuelo. No olvidemos la posibilidad de hacer tomas diferentes a las previstas por incidencias tácticas o averías.

\section{AERONAVES IMPLICADAS}

Como norma general, la aeronave empleada en las aeroevacuaciones avanzadas es el helicóptero HD-21 «Superpuma» de Ejército del Aire, perteneciente al destacamento HELISAF (Figura 1). Como alternativa para este tipo de misiones, contamos con el helicóptero $\mathrm{CH}-47$ «Chinook» o los «Cougar» del Ejército de Tierra ${ }^{5,6}$, que pertenecen al destacamento ASPUHEL (Tabla 1).

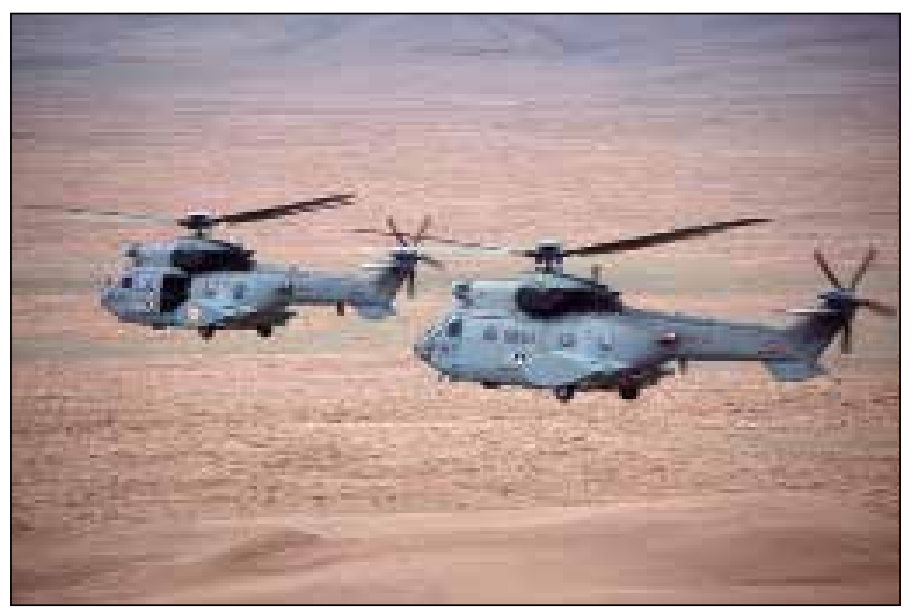

Figura 1. Helicóptero HD-21 «Superpuma» del Ejército del Aire. 
Tabla 1. Capacidades de los medios de ala rotatoria habituales.

\begin{tabular}{|ll|}
\hline \multicolumn{1}{|c|}{ Aeronave } & \multicolumn{1}{c|}{ Capacidad } \\
\hline Superpuma HD-21 & 2 camillas, 5 bajas ambulatorias \\
Chinook $\mathrm{CH}-47$ & 24 camillas en configuración de bajas masivas \\
\hline
\end{tabular}

En las aeroevacuaciones tácticas, empleamos medios de ala fija tales como el CASA 295 y el C-130 «Hércules» del Ejército del Aire integrados en los destacamentos $A L C O R$ y MIZAR respectivamente ${ }^{7,8}$, aunque el destacamento ALCOR ya ha replegado.

Por razones logísticas en ocasiones puntuales se han empleado otros medios de ala fija para este tipo de evacuaciones, como el C-160 «Transalp» alemán.

\section{TRIPULACIÓN SANITARIA}

Existen dos tripulaciones sanitarias constituidas cada una por un Oficial Médico de vuelo, un Oficial Enfermero y un sanitario MPTM. El Oficial Médico y el Sanitario MPTM están encuadrados en la plantilla del Hospital ROLE 2E, mientras que el Oficial Enfermero depende orgánicamente del destacamento HELISAF.

Los tres, junto al resto de material asistencial de a bordo y dispositivos de electromedicina, forman el equipo necesario para alcanzar la capacidad de asistencia en soporte vital avanzado.

Generalmente una tripulación sanitaria permanece en alerta y la otra en retén en turnos de 24 horas, aunque en casos de bajas masivas o de dos pacientes críticos, se pueden activar las dos tripulaciones.

\section{HABILIDADES ASISTENCIALES}

La casuística acumulada durante estos años, ha ido definiendo los requerimientos asistenciales en función de los pacientes atendidos, el medio utilizado y el ambiente táctico. Abarca todo el abanico propio de la medicina de urgencias y emergencias, con especial mención de la enfermedad traumática en un entorno bélico con heridos por arma de fuego y explosivos, además de bajas masivas, niños y lactantes. Por motivos de seguridad, la asistencia se realiza habitualmente en vuelo,

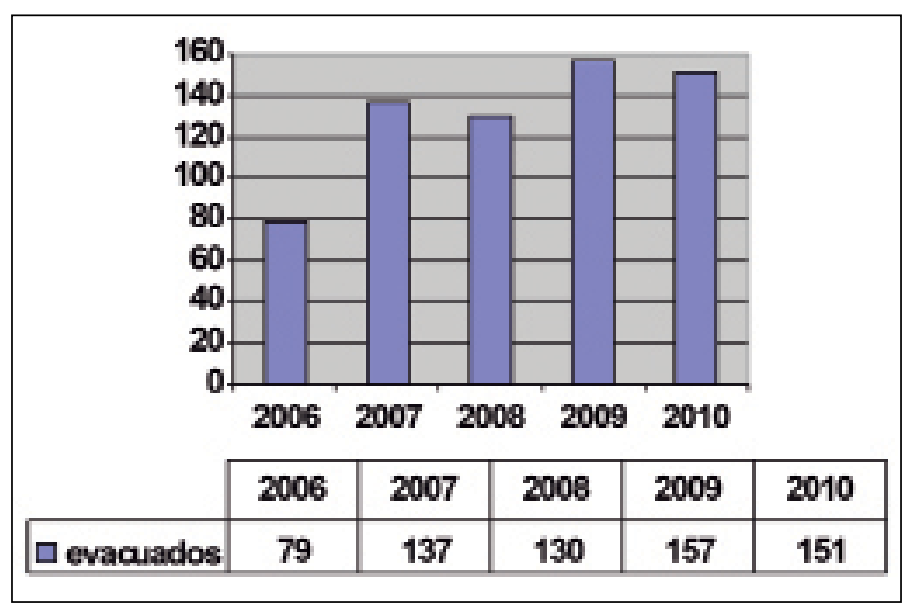

Figura 2. Evolución anual del número de evacuaciones realizadas por el equipo MEDEVAC español (registro series UMAAD MADRID). con puertas abiertas, expuestos al viento, a las condiciones climáticas y frecuentemente en la oscuridad.

Es imprescindible la formación, entrenamiento y aplicación de conocimientos y técnicas en urgencias y emergencias, soporte vital avanzado cardiológico y traumatológico en combate (soporte vital avanzado de combate), asistencia inicial al trauma a nivel prehospitalario o la asistencia a la bajas tácticas de combate (tactical combat casualty care), con las consideraciones propias de ambiente aeronáutico para garantizar buenos resultados a través de los cursos de medicina y enfermería de vuelo, aeroevacuación sanitaria además de entrenamiento con aeronaves con vuelos reales.

Tabla 2. Características de las bajas en combate atendidas en Afganistán entre los años 2006 y 2008 por los efectivos de la Sanidad Militar española.

\begin{tabular}{|c|c|c|c|}
\hline & Variable & $\mathbf{n}$ & $\%$ \\
\hline \multirow[t]{2}{*}{ Sexo } & Varón & 246 & 96 \\
\hline & Mujer & 10 & 4 \\
\hline \multirow[t]{2}{*}{ Edad } & 25-29 años & 101 & 39 \\
\hline & Otros & 155 & 61 \\
\hline \multirow[t]{3}{*}{ Grupo de pertenencia } & Afghan National Army & 112 & 44 \\
\hline & ISAF & 62 & 24 \\
\hline & Civil & 82 & 32 \\
\hline \multirow[t]{2}{*}{ Medidas de protección } & $\mathrm{Si}$ & 62 & 25 \\
\hline & No & 194 & 75 \\
\hline \multirow[t]{4}{*}{ Localización } & Norte & 54 & 21 \\
\hline & Sur & 134 & 53 \\
\hline & Este & 6 & 2 \\
\hline & Oeste & 62 & 24 \\
\hline \multirow[t]{3}{*}{ Evolución temporal } & 2006 & 49 & 19 \\
\hline & 2007 & 92 & 36 \\
\hline & 2008 & 114 & 44 \\
\hline \multirow[t]{4}{*}{ Estación del año } & Primavera & 31 & 12 \\
\hline & Verano & 93 & 36 \\
\hline & Otoño & 85 & 33 \\
\hline & Invierno & 47 & 19 \\
\hline \multirow[t]{2}{*}{ Agente lesional } & Explosivo & 183 & 71 \\
\hline & Arma de fuego & 73 & 29 \\
\hline \multirow[t]{6}{*}{ Área afectada* } & Miembros inferiores & 123 & 48 \\
\hline & Miembros superiores & 99 & 39 \\
\hline & Abdomen & 56 & 22 \\
\hline & Tórax & 49 & 19 \\
\hline & Cuello & 30 & 12 \\
\hline & Cabeza & 11 & 4 \\
\hline \multirow[t]{6}{*}{ N. ${ }^{\circ}$ Áreas afectadas } & 1 Área & 142 & 55 \\
\hline & 2 Áreas & 59 & 23 \\
\hline & 3 Áreas & 39 & 15 \\
\hline & 4 Áreas & 13 & 5,5 \\
\hline & 5 Áreas & 1 & 0,3 \\
\hline & 6 Áreas & 2 & 0,6 \\
\hline
\end{tabular}

* Relacionamos esta variable con la de número de áreas afectadas, ya que el $45 \%$ de las mismas presentan lesiones en más de una zona anatómica. 
Muchos de los modelos formativos descritos son reconocidos en la esfera internacional y se aplican hoy día en Afganistán. Es fundamental por lo tanto su conocimiento y aplicación por parte de todo el personal sanitario, para permitir una correcta labor asistencial en el seno de una fuerza multinacional y alcanzar la más elevada coordinación y eficacia sobre un paciente que no admite demoras.

\section{PERFIL DE LA BAJA EVACUADA}

Según el estudio de Navarro9 ${ }^{9}$ las bajas atendidas en la región oeste de Afganistán por miembros del Cuerpo Militar de Sanidad español entre el año 2006 y 2008 siguen el perfil que se muestra en la Tabla 2. De las 256 bajas en combate recogidas en su muestra, 194 (76\%) fueron evacuadas al ROLE 2E español empleando medios aéreos (Figura 2).

\section{RESUMEN FINAL Y PERSPECTIVAS DE FUTURO}

La experiencia ha demostrado las ventajas de la evacuación sanitaria mediante aeronaves, especialmente la rapidez en la asistencia inicial y en el traslado. El modelo de equipo asistencial español, compuesto por médico enfermero y sanitario, adecuadamente formado y entrenado, se considera mejor que el de otros países aliados al permitir realizar un soporte vital avanzado sobre la baja, desde la misma transferencia a la aeronave.

Más y mejor asistencia en el menor tiempo posible desde el punto de impacto.

La especial gravedad del paciente por enfermedad traumática en el ambiente táctico, orienta a desarrollar conceptos relacionados con el control de daños o el empleo de medios de diagnóstico precoz, con el objetivo de aumentar al máximo las capacidades asistenciales de un equipo sanitario completo.

La experiencia acumulada por la fuerza multinacional desplegada en Afganistán ratifica la importancia en la normalización de procedimientos asistenciales, junto con un adecuado entrenamiento de los mismos.

La creación y consolidación de un cuerpo doctrinal en Urgencias y Emergencias con formación inicial y continuada, entrenamiento táctico-asistencial y la indispensable normalización multinacional, constituye la hoja de ruta que permita la máxima calidad asistencial adaptada a cada situación.

\section{BIBLIOGRAFÍA}

1. Navarro R, Bartolomé E, Jara I, Oreja A, González G. Capacidades y asistencia sanitaria realizada por el ROLE-2 español en la FSB de Herat (Afganistán) desde febrero a julio del 2007. Sanid. Mil. 2008; 64 (2): 98-104.

2. Maimir F, Hernández A. Metodología y material en el transporte sanitario militar en área de operaciones. Med. Mil. 2006; 62 (1): 32-38.

3. Betegón A. Evacuación sanitaria en área/zona de operaciones. Med. Mil. 2006; 62 (3): 159-161.

4. Bartolomé E, Navarro R. Traumatismo craneoencefálico abierto por impacto directo de arma de fusilería ligera atendido en el ROLE 2. Sanidad Militar 2007; 3 (4): $305-8$

5. García J. El servicio de sanidad en las operaciones aeromóviles. Medicina Militar 1985; 41 (4): 441-3.

6. Hernando AE, Estella JF, Perales N, Coma R, Mateos J. Evacuación aérea sanitaria en el Ejército. Med. Mil. 1985; 41 (2): 214-221.

7. Pérez V, Laguardia JC. Aviocar C-212. Empleo sanitario. Med. Mil. 1987; 43 (6): 621-625.

8. Laguardia J.C, Pérez V. C-130 Hércules. Empleo sanitario. Med. Mil. 1987; 43 (5): 493-498.

9. Navarro R. Bajas por arma de fuego y explosivos. Experiencia del Hospital Militar español desplegado en Herat (Afganistán) 2005-2008 [tesis doctoral]. Madrid: Universidad Autónoma de Madrid; 2009. 